\title{
6. Dichtebestimmungen
}

von gesattigten Dampfen und Flussigkeiten; von Rudolf Freinerr von Hirsch.

(Hleren Taf. VII.)

Einleitung.

Die specifischen Volumina der gesăttigten Dämpfe und der Flüssigkeiten unter Sättigungsdruck spielen einestheils in den Gleichungen der Thermodynamik eine Rolle, andererseits scheinen sie geeignet, über den Zusammenhang des flüssigen und dampfförmigen Aggregatzustandes wichtige Aufschlüsse zu geben.

Dennoch umfasst die experimentelle Untersuchung verhältnissmässig wenige Stoffe und ist auch für diese nur innerhalb enger Temperaturgrenzen ausgeführt; besonders für hohe Temperaturen sind die vorliegenden Resultate so wenig zahlreich, dass weitere Bestimmungen als wünschenswerth erschienen.

Bei der Ausführung der Versuche bediente ich mich folgender von S. Young angegebenen Methode ${ }^{1}$ ): Eine beiderseitig geschlossene Glasröhre wird nacheinander mit zwei verschiedenen Gewichtsmengen $p$ und $p^{\prime}$ der zu untersuchenden Substanz gefüllt ${ }^{2}$ ) und auf eine constante Temperatur $T$ ge. bracht. Aus dem abgelesenen Stand des Meniscus ergeben sich in dem calibrirten Rohr die von Flussigkeit und Dampf eingenommenen Volumina, $V_{a}$ bez. $V_{b}$ für die erste, $V_{a}^{\prime}$ bez. $\Gamma_{b}^{\prime}$ für die $z$ weite Füllung. Bildet man die Grössen

$$
\begin{array}{ll}
S a=\frac{V a}{p}, & S b=\frac{V b}{p} . \\
S a^{\prime}=\frac{V^{\prime} a}{p^{\prime}}, & S b=\frac{V^{\prime} b}{p^{\prime}},
\end{array}
$$

1) Eine Uebersicht und Discussion der sonst bekannten Methoden findet sich in der Dissertation.

2) S. Young, Journ. of the Chemical Society of London 59. p. 911 ff. 1891. 
so ist $S_{b}$ bei der ersten Fullung das aus der Gewichtseinheit entwickelte Dampfvolumen, $S_{a}$ die von der Gewichtseinheit ubrig bleibende Flüssigkeit; also das specifische Volumen der Flüssigkeit, d. h. das Volumen der Gewichtseinheit Flüssigkeit

$$
S=S_{a}+S_{b_{i} r},
$$

wo $r$ das Verbältniss der specifischen Volumina von Dampf und Flüssigkeit bedeutet. Dieselbe Betrachtung, auf die zweite Füllung angewendet, ergiebt

$$
S=S_{a}^{\prime}+S_{r_{r}} .
$$

Aus beiden Gleichungen berechnet sich

$$
\begin{aligned}
& S=\frac{S_{a} S_{b}^{\prime}-S_{u^{\prime}} S_{i}}{S_{b}^{\prime}-S_{b}}, \\
& r=\frac{S_{b}}{S-S_{u}}=\frac{S_{i}^{\prime}}{S-S_{a}^{\prime}}
\end{aligned}
$$

und

$$
s=r . S \text {. }
$$

Der bei den Versuchen benutzte Apparat war bis auf einige geringfügige Aenderungen nach den Angaben von S. Young ') angefertigt (vgl. Fig. 1):

Ein vertical stehendes, etwa $20 \mathrm{~mm}$ weites, $60 \mathrm{~cm}$ langes Rohr aus dickwandigem, schwer schmelzharem Glase (Verbrennungsrohr $a b$ ) war in einen kurzen Messing. ansatz $(b c)$ eingekittet; derselbe trug 2 wei seitliche Ansatzrobre, von denen das eine $z \mathfrak{u}$ einer Kuhlschlange $k$, das andere $z \mathfrak{u}$ einem Kolben fübrte, der die zur Herstellung der hohen Temperaturen dienenden Siedeflüssig. keiten enthielt. Mit einem

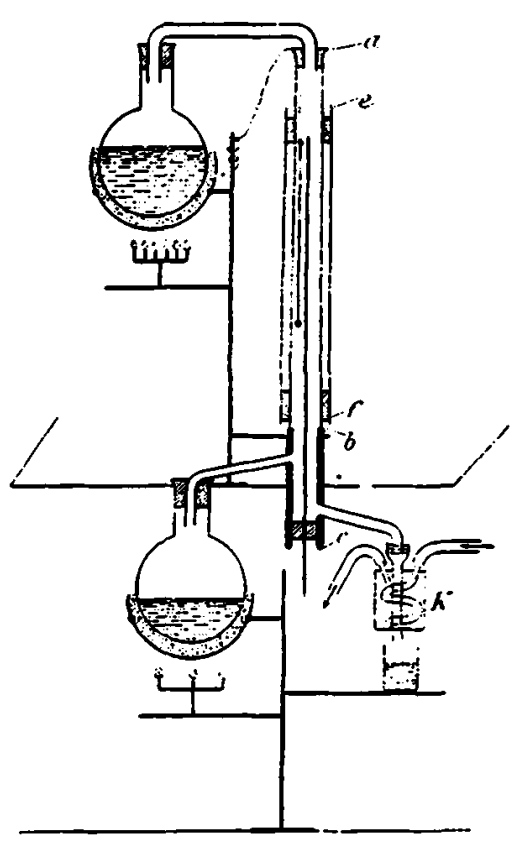

Fig. 1.

1) S. Young, Joum. Chen. Soc. 59. p. 37. 1891. 
ebensolchen Kolben war das obere Ende der Röhre al durch ein doppelt gebogenes Messingrohr verbunden. Die Röhren sind in die Siedekolben mittels durchbohrter Korke eingesetzt; diese stehen in Sandbädern und werden durch starke Bunsenbrenner erhitzt. Das Dampfrohr $a b$ ist von einem weiteren Schutzrohr ef umgeben, welches durch Korke auf diesem gehalten wird. Ausserdem sind, ebenfalls zum Schutz gegen Abkühlung nach aussen, alle erwärmten Theile des Apparates mit dickem Asbestpapier umwickelt. Das Versuchsrohr wurde anfangs nach den Angaben von S. Young an einem Stiel von unten in das Dampfrohr eingeführt und mittels eines durchbohrten Korkes festgeklemmt, während ein Thermometer von oben an einem Draht in den Dampfraum hing. Da jedoch der Kork beständig undicht wurde und die Röhre nicht ordentlich festhielt, so wurde später die untere Oeffnung des Dampfrohres ganz verscblossen, die Versuchsröhre aber obne Stiel an das Thermometer gebunden von oben eingeführt. Gegenüber der Flüssigkeitsoberfläche $o$ ist aus der Asbestumwickelung vorne und hinten ein längliches Fenster ausgeschnitten; das eine dient zur Beleuchtung des Meniscus mit einer kleinen Glüblampe, das andere zur Ablesung durch ein etwa $5 \mathrm{~m}$ entferntes Fernrohr. Da während der Versuche die Drucke im Rohr sehr gross werden, so ist zum Schutz gegen etwaige Explosionen der ganze Apparat von einem Drahtgitter umgeben. Der Gang der Versuche bedarf keiner Erläuterung. Bringt man die Flüssigkeit in den Kolben zum Sieden, so erfüllt sich der Inneurauı des Apparates mit einem Dampfstrom von constanter Temperatur; diese sowie der Stand des Flüssigkeitsmeniscus in der Röhre werden abgelesen. Der verbrauchte Dampf strömt durch die von kaltem Wasser umflossene Kühlschlange $a b$ und wird in einem unterstellten Gefäss als lauwarme Flüssigkeit wiedergewonnen, welche sofort zur Wiederholung des Versuches benutzt werden kann.

Die Füllung der Versuchsröhren mit den zu untersuchenden Flüssigkeiten geschah nach der von Young angegebenen Methode. ${ }^{1}$ ) Ein Glasrohr von $2^{1 / 2}$ bis $5 \mathrm{~mm}$ innerem Durch-

1) S. Young, Journ. Chem. Soc. 59. p. 37 ff. 1891. 
messer und etwa $5 \mathrm{~mm}$ Wandstärke (vgl. Fig. 2) wurde mit einer Millimetertheilung versehen und mittels Quecksilberfadens calib. rirt. Hierauf wurde die Röhre etwa $30 \mathrm{~cm}$ von einem Ende zugeschmolzen (bei $a$ ), sodass das

Stück $a b$ den Stiel bildete; bei den späteren Versuchen, wo derselbe weggelassen wurde,

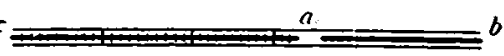

Fig. 2. zog man die Röhre bei $a$ ab. Das Ende bei $a$ wurde mit Quecksilber ausgewogen und auf das sorgfältig gereinigte Rohr bei $c$ der nebenbei abgebildete Ansatz aufgeschmolzen. Nachdem der ausgebauchte Theil desselben etwa zur Hälfte mit der zu prüfenden Flüssigkeit gefült war, wurde die Oeffnung $o$ mit einer Quecksilberpumpe verbunden und das Rohr ausgepumpt. Befindet sich die Oeffnumg des kleinen Rohrstückes $r$ unter der Oberfläche der Flüssigkeit, so saugt es dieselbe jetzt eili, ist eine genügende Quantität eingedrungen, so dreht man das Rohr, sodass die Oeffnung ron $r$ jetzt über dem Niveau der Fliissigkeit steht und die Saugewirkung aufhört. Nach einigen Stössen der Luftpumpe wird sodann das Ansatzstück an der zu diesem $Z$ weck verengten Stelle $e$ abgeschmolzen. Nachdem man der Flüssigkeit einige Stunden Zeit gelassen hat, an den Wänden herabzufliessen,

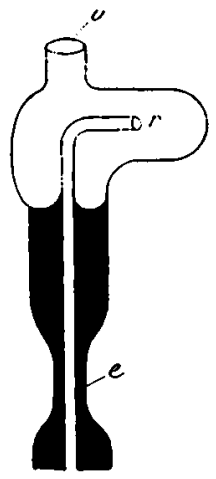

Fig. 3. wird der Stand bei Zimmertemperatur abgelesen und hieraus mittels des bekannten specifischen Gewichtes das Gewicht der Füllung berechnet. Hierauf wird die Röhre umgekehrt und jetzt mittels des bekannten Gewichtes der Füllung das Volumen des anderen Endes bestimmt. Diese Methode der Füllung ist nicht gerade sehr einfach, dafür leistet sie aber Gewähr, dass die Luft aus der Röhre vollkommen entfernt ist, und die Substanz, welche ja nur mit den Rohrwänder in Berührung kommt, nicht verunreinigt wird (vgl. Fig. 3).

Als Siedeflüssigkeiten wurden folgende Substanzen verwendet:

Dimethylanilin

Diäthylanilin

Benzoesäureisobutylester

Bromnaphtalin
(Siedepunkt 190 )

(Siedepunkt $\mathbf{2 1 2}^{\circ}$ )

(Siedepunkt 237\%)

(Siedepunkt $276^{\circ}$ ) 
Die drei ersten Substanzen sieden sehr constant, Brom. naphtalin dagegen zersetzt sich sehr stark, sodass die Temperatur während eines Versuches nie ganz constant wird, besonders wenn man den in den Kolben bleibenden Rest der Flüssigkeit nicht jedesmal entfernt und nur das Destillat verwendet; thut man dies, so braucht man sebr grosse Mengen Substanz; auch der sehrunangenehme Geruch des Bromnaphtalins, der ein längeres Arbeiten in diesen Dämpfen erschwert, lässt es als Siedeffüssigkeit wenig geeignet erscheinen.

Betreff's der Berechnung der Versuche ist folgendes zu erwähnen :

Alle Volumina sind auf $1 / 10 \mathrm{cmm}$ genau bestimmt und hieraus die Grössen $S_{a}$ und $S_{b}$ auf 4 Decimalstellen ansgerechnet; die vierte Stelle, welche keinen Anspruch auf Richtigkeit machen kann, ist in dem nach der Formel

$$
S=\frac{S_{u} S_{i}^{\prime}-S_{u}{ }^{\prime} S_{l}}{S_{b}^{\prime}-S_{l}}
$$

berechneten Werth des specifischen Volumens weggelassen. Fin immerhin möglicher Ablesefehler von $1 / 10 \mathrm{~mm}$ macht sich bereits in der dritten Decimale bemerkbar, deren Einheiten daher nicht als sicher betrachtet werden können.

Die Berechnung von

$$
r=\frac{S_{i}}{S-S_{a}}
$$

wird um so ungenauer, je kleiner $S-S_{a}$ ist, d. h. je weniger Substanz verdampft ist. Die Methode ist daher nur für Temperaturen erheblich über dem normalen Siedepunkte der $z \mathbf{u}$ untersuchenden Substanz anwendbar.

Wird $S$ zu gross gefunden, so muss $r$ zu klein ausfallen und umgekehrt. Das specifische Volumen des Dampfes, auf das es eigentlich ankommt, $s=r . S$, ist daher immer weniger ungenau als $r$.

Es wurden folgende Correctionen angebracht.

1. Correction wegen des Caliberfehlers des Rohres.

2. Correction wegen des Meniscus (stets als $1 / 3$ Grundfläche-Höhe berechnet). Dieselbe Correction wurde auch bei der Calibrirung mit Quecksilber angewendet. 
3. Eine Correction wegen Ausdebnung des Glases war nicht nöthig, da dieselbe auch bei den grössten Werthen der Volumina $(2000 \mathrm{cmm})$ mit dem gewöhnlichen Ausdebnungscoefficienten des Glases 0,000025 , erst $0,05 \mathrm{cmm}$ ausmacht, während alle Volumina nur auf $0,1 \mathrm{cmm}$ berechnet sind.

4. Die wichtigste Correction ist die wegen des bei Zimmertemperatur im Rohr vorbandenen Dampfes, welche bei der Gewichtsbestimmung einige Milligramm ausmachen kann. Sie beeinflusst den Werth von $S$ fast gar nicht, den von $r$ sehr stark, um so stärker je grösser $r$, also je niedriger die Temperatur ist.

Bei dieser Correction wurde für den Dampf stets die normale Dichte angenommen, d. h. das halbe Moleculargewicht multiplicirt mit der Dichte des Wasserstoffs, 0,0008988.

Alle Versuche wurden mindestens dreimal, und wenn die Resultate schlecht übereinstimmten, noch öfter wiederholt.

Um $S$ aus den Versuchen mehrere Nale zu berechnen, müssen mindestens drei verschiedene Füllungen untersucht werden, wie dies bei der einen Hälfte der geprüften Substanzen auch geschah; da aber in der Gleichung

$$
S=\frac{S_{a} S_{b}^{\prime}-S_{a}^{\prime} S_{b}}{S_{\iota}^{\prime}-S_{b}}
$$

$S_{b}-S_{\imath}$ nicht $2 u$ klein werden darf, so können nur solche Werthe zur Berechnung combinirt werden, bei welchen die Füllungen erheblich voneinander abweichen. $\mathrm{Da}$ die mehrmals berechneten Werthe von $S$ stets gut miteinander übereinstimmten, so erscheint auch da, wo diese Grösse aus den Versuchen nur einmal berechnet werden konnte, die Genauigkeit hinreichend.

\section{Versuchsresultate.}

Es wurden folgende Substanzen untersucht: Toluol; Ortho-, Para- und Metaxylol; Propionsäure; normale und Isobuttersäure ${ }^{1}$ ).

1) Ueber Reinigung der Substanzen vgl. die Dissertation. 
I. Toluol.

Siedepunkt $110^{\circ}$; specifisches Gewicht bei $18^{\circ}$ : 0,8665 .

Die Resultate sind in den folgenden Tabellea zusammengestellt $\left.{ }^{1}\right)$ :

Tabelle 1.

\begin{tabular}{|c|c|c|c|c|c|c|c|c|}
\hline \multirow{2}{*}{$T$} & \multicolumn{2}{|c|}{1} & \multicolumn{2}{|c|}{2} & \multicolumn{2}{|c|}{3} & \multicolumn{2}{|c|}{4} \\
\hline & $S_{n}$ & $S_{k}$ & $S_{a}$ & $S_{b}$ & $S_{t}$ & $S_{b}$ & $S_{a}$ & $S_{t}$ \\
\hline $190^{\circ}$ & 1.2318 & 7,0657 & & & 1,2255 & 7,1188 & 1,3476 & 3,3628 \\
\hline 212 & 1,1949 & 7,1026 & & & 1,1751 & 7,1692 & 1,3648 & 3,3456 \\
\hline 237 & & & 1,4318 & 2,5276 & 1,0798 & 7,2645 & 1,3634 & 3,3470 \\
\hline 276 & 0,6685 & 7,6290 & 1,4558 & 2,5036 & & & 1,3207 & 3,3897 \\
\hline
\end{tabular}

Tabelle 2.

\begin{tabular}{|c|c|c|c|c|c|c|c|c|}
\hline \multirow{2}{*}{$T$} & \multicolumn{4}{|c|}{ I } & \multicolumn{4}{|c|}{ II } \\
\hline & $S$ & $r$ & $s$ & & $S$ & $r$ & $s$ & \\
\hline $190^{\circ}$ & 1,452 & 30,7 & 44,7 & 1.4 & 1,456 & 31,4 & 45,7 & 3.4 \\
\hline 212 & 1,516 & 22,1 & 33,5 & 1.4 & 1,534 & 19,8 & 30,4 & 3.4 \\
\hline 237 & 1,620 & 13,4 & 21,8 & 2.3 & 1,606 & 138 & 22.1 & 3. 4 \\
\hline 276 & 1,839 & 6,43 & 11,8 & 1.4 & 1,842 & 6,59 & 12,4 & 1.2 \\
\hline
\end{tabular}

Tabelle 3.

\begin{tabular}{c||c||c||c||c||c}
\hline \hline$T$ & $S$ & $r$ & $s$ & $D$ & $d$ \\
\hline \hline $190^{\circ}$ & 1,455 & 31,1 & 45,2 & 0,687 & 0,022 \\
212 & 1,525 & 21,0 & 32,0 & 0,656 & 0,031 \\
237 & 1,613 & 13,6 & 22,0 & 0,620 & 0,045 \\
276 & 1,841 & 6,51 & 12,1 & 0,543 & 0,082
\end{tabular}

Die erste Tabelle enthält die aus der Beobachtung an vier Versuchsreihen entnommenen Werthe von $S_{a}$ und $S_{b}$, die zweite die hieraus berechneten Grössen $S, r$ und $s$, wobei die letzte Columne die Nummern der Beobachtungsreihen angiebt, welche der Berechnung $\mathrm{zu}$ Grunde liegen. In der dritten Tabelle

1) Bei Toluol ist das ganze Material angegeben, bei den folgenden Substanzen nur die für Temperaturintervalle von $10^{\circ}$ abgeleiteten Werthe von $S, s, D$ und $d$. 
sind die aus beiden Berechnungen gezogenen Mittelwerthe ron $S ; r$ und $s$, sowie deren reciproke Werthe, die Dichten $D=1 / S$ und $d=1 / s$ zusammengestellt. Trägt man diese Werthe $D$ und $d$ in einem Coordinatensystem, dessen Ordinaten die Temperaturen sind, als Abscissen auf, so sollen nach einem ron Matthias aufgestellten Gesetz $\left.{ }^{1}\right)$ die Mittelwerthe $(D+d) / 2$ auf einer Geraden, dem sogenannten Durchmesser der Dichtecurven, liegen. Dieses Gesetz zeigte sich hier wie bei allen anderen untersuchten Substanzen vollständig bestätigt, wie nachstehende Tabelle zeigt, in welcher die erste Columne die beobachteten, die zweite die nach der Formel

$$
\Delta=0,4464-0,000483 t
$$

berechneten Werthe von $(d+D) / 2$ zeigt:

Tabelle 4.

\begin{tabular}{l||l||l}
\hline $190^{\circ}$ & $0,35 \cdot 17$ & 0,3546 \\
212 & 0,3435 \\
237 & 0,3328 & 0,3440 \\
276 & 0,3128 & 0,3319 \\
\end{tabular}

Da also die Curve, welche die Dichten als Function der Temperatur darstellt, einen geradlinigen Durchmesser hat, so ist die Annahme naheliegend, dass die Curve eine Parabel sei; thatsächlich haben Cailletet und Matthias') für eine Reihe von Substanzen die Formel $d=\alpha-\beta t-\gamma \sqrt{\overline{4}-t}$ bestätigt gefunden, wobei sie allerdings für den der Flüssigkeit entsprechenden Curvenast andere Constanten $\alpha, \beta, \gamma$ annahmen als für den Theil, welcher dem Dampf entspricht; ( $q$ bedeutet die kritische Temperatur). Um diese Gesetzmässigkeit zu prüfen, wurden die Constanten der Parabel $y^{2}=m x+n y$ bestimmt. welche auf ein schiefwinkliges Coordinatensystem bezogen ist, dessen eine Axe dem Durchmesser $\Delta=a-t \operatorname{tg} a$ parallel ist, sodass

$$
y=d+t \operatorname{tg} \alpha, \quad x=\frac{t}{\cos a}
$$

1) E. Mathias, Compt. reud. 115. p. 35.1890.

2) L. Cailletet u. E. Matthias, Compt. rend. 10\%. p. 1563. 1887. 
ist. Die Scheitelcoordinaten dieser Parabel sind

$$
\frac{n}{2}=a \text { und }-\frac{n^{2}}{4 m}=\frac{y}{\cos \alpha}
$$

( 9 kritische Temperatur); also $n=2 a=0,8928$

$$
m^{\prime}=\frac{m}{\cos a}=\frac{y^{2}-n y}{t} .
$$

Setzt man in dieser Gleichung nacheinander die vier beobachteten Werthe von $d$ bez. $D$ ein, so erbält man

$$
\begin{aligned}
& m^{\prime}=0,0004680=0,0004659 \\
& m^{\prime}=0,0004804=0,0004820 \\
& m^{\prime}=0,0004959=0,0004904 \\
& m^{\prime}=0,0005293=0,0005302 .
\end{aligned}
$$

Die Werthe der Constante werden also immer grösser, wenn man von niedrigen zu höheren Temperaturen fortschreitet, was auf eine regelmässige Abweichung von der Parabelgestalt deutet. Diese Abweichung ist sehr bedeutend; denn berechnet man mit dem Mittelwerth von $m^{\prime}=0,0004932$ die kritische Temperatur $\vartheta=-\left(n^{2} / 4 m^{\prime}\right)$, so findet man $452^{\circ}$ anstatt des beobachteten ${ }^{1}$ ) Werthes $320^{\circ}$. Die Curve, welche die Dichten als Function der Temperatur darstellt, ist also im Vergleich mit dieser Parabel erheblich abgeplattet; sie wurde aus den beobachteten Werthen $D$ und $d$ auf graphischem Wege für das Intervall $190^{\circ}$ bis $280^{\circ}$ construirt. Die aus der Curve abgelesenen Werthe von $D$ und $d$ und ihre reciproken Werthe $S$ und $s$ sind in beistehender Tabelle für Intervalle von $10^{\circ}$

\begin{tabular}{|c|c|c|c|c|c|c|c|c|c|}
\hline$T$ & $D$ & $d$ & $S$ & $s$ & $T$ & $D$ & $d$ & $S$ & $s$ \\
\hline 190 & 8 & 022 & 1,455 & 45,5 & 240 & 0,614 & 0,048 & 1,629 & 20,8 \\
\hline 200 & 0,672 & 0,026 & 1,488 & 38,5 & 250 & 0,594 & 0,057 & 1,684 & 17,5 \\
\hline 210 & 0,658 & 0,030 & 1,520 & 33,3 & 260 & 0,574 & 0,066 & 1,742 & 15,1 \\
\hline 220 & 0,644 & 0,035 & $1, \mathbf{5 5 3}$ & 28,6 & 270 & 0,554 & 0,076 & 1,805 & 13,2 \\
\hline 230 & 0,630 & 0,040 & 1,587 & 25,0 & 280 & 0,534 & 0,055 & 1,873 & 11,8 \\
\hline
\end{tabular}
angegeben; die Curve selbst ist beigefügt (vgl. Taf. VII). In einer zweiten Tabelle sind die aus der Curve entnommenen Werthe von $s$ und $s$ mit den aus der Beobachtung direct abgeleiteten rerglichen.

Tabelle $\overline{\mathbf{5}}$.

1) Pawlewski, Chem. Ber. 21. p. 2141. 1888. 
Dichte gesättigter Dämpfe und Flüssigktiten.

Tabelle 6.

\begin{tabular}{c||c|c|cc}
\hline \multirow{2}{*}{$T$} & \multicolumn{2}{|c|}{ Beobachtet } & \multicolumn{2}{|c}{ Aus der Curve } \\
\hline & $S$ & $s$ & $S$ & $s$ \\
\hline 190 & 1,455 & 45,2 & 1,455 & 45,5 \\
212 & 1,525 & 32,0 & 1,527 & 32,4 \\
237 & 1,613 & 22,0 & 1,616 & 22,1 \\
276 & 1,841 & 12,1 & 1,346 & 12,3
\end{tabular}

II. Orthosylol.

Siedepunkt 141"; specifisches Gewicht bei 18": 0,8790 . Es wurde wieder die Curve der Dichten construirt, deren Durchmesser der Gleichung

$$
\Delta=0,4578-0,000481 t
$$

entspricht. Die nach dieser Formel berechneten Werthe sind in Tab. 8 mit den aus der Beobachtung sich ergebenden Grössen $(d+D) / 2$ verglichen und zeigen die Richtigkeit des Mattbias'schen Gesetzes für Orthoxylol. Aus der Curve sind die Werthe $d$ und $D$ für Intervalle von $10^{\circ}$ abgelesen und die zugehörigen Grössen $s$ und $S$ berechnet (Tab. 7).

Tabelle 7.

\begin{tabular}{|c|c|c|c|c|c|c|c|c|c|}
\hline$T$ & $S$ & $s$ & $D$ & $d$ & $r$ & $s$ & $s$ & $y$ & $d$ \\
\hline 19 & 1,396 & 71,4 & 0.716 & 0,014 & 240 & 1,5 & $\mathbf{3 5}, 7$ & 0,656 & 0,028 \\
\hline 200 & $1,41 \mathrm{y}$ & 62,5 & 0,705 & 0,016 & 250 & 1,560 & 29,4 & $0,6.11$ & 0,034 \\
\hline 210 & $1, \mathbf{4 4 1}$ & 52,6 & 0,694 & 0,019 & 260 & 1,600 & 25,0 & 0,625 & 0,040 \\
\hline 220 & 1,466 & 47,6 & 0,682 & 0,021 & 270 & 1,642 & 21,7 & 0,609 & 0,046 \\
\hline 230 & 1,493 & 41,7 & 0,670 & 0,024 & 280 & 1,686 & 19,2 & 0,593 & 0,052 \\
\hline
\end{tabular}

Tabelle 8.

\begin{tabular}{c|c|c}
\hline$T$ & $\frac{d+D}{2}$ & $\Delta$ \\
\hline 190 & 0,3663 & 0,3664 \\
212 & 0,3546 & 0,3558 \\
237 & 0,3459 & 0,3438 \\
276 & 0,3249 & 0,3250
\end{tabular}

Ann. d. Phys. u. Chem. N. F. 69. 
III. Paraxylol.

Siedepunkt $137^{\circ}$; Schmelzpunkt $13,0^{\circ}$; specifisches Gewicht bei $18^{\circ}$ : 0,8620 .

Der Durchmesser entspricht der Gleichung

$$
\begin{gathered}
\Delta=0,3902-0,00344 t . \\
\text { Tabelle } 9 .
\end{gathered}
$$

\begin{tabular}{c||c||c}
\hline \hline$T$ & $\frac{d+D}{2}$ & $\Delta$ \\
\hline \hline $190^{\circ}$ & 0,3237 & 0,3248 \\
212 & 0,3163 & 0,3172 \\
237 & 0,3127 & 0,3086 \\
276 & 0,2933 & 0,2952
\end{tabular}

Tabelle 10.

\begin{tabular}{c||c||c||c||c||c||c||c|c|c}
\hline \hline$T$ & $D$ & $d$ & $S$ & $s$ & $T$ & $D$ & $d$ & $S$ & $s$ \\
\hline \hline $190^{\circ}$ & 0,620 & 0,028 & 1,613 & 35,7 & $240^{\circ}$ & 0,575 & 0,041 & 1,739 & 24,4 \\
200 & 0,612 & 0,030 & 1,634 & $\mathbf{3 3 , 3}$ & 250 & 0,562 & 0,045 & 1,779 & 22,2 \\
210 & 0,603 & 0,032 & 1,658 & $\mathbf{3 1 , 3}$ & $\mathbf{2 6 0}$ & 0,548 & 0,051 & 1,525 & 19,6 \\
220 & 0,594 & 0,035 & 1,684 & 28,6 & 270 & 0,534 & 0,057 & 1,873 & 17,5 \\
230 & 0,085 & 0,038 & 1,710 & $\mathbf{2 6 , 3}$ & 280 & 0,520 & 0,062 & 1,923 & 16,1
\end{tabular}

IV. Metaxylol.

Siedepunkt $138^{\circ}$; specifisches Gewicht bei $18^{\circ}$ : 0,8667 . Die Durchmessergleichung wird

$$
\Delta=0,4385-0,000438 t \text {. }
$$

Tabelle 11 .

\begin{tabular}{c||c|c}
\hline$T$ & $\frac{D+d}{2}$ & $\Delta$ \\
\hline \hline 190 & 0,3552 & 0,3553 \\
212 & 0,3457 & 0,3457 \\
237 & 0,3351 & 0,3348 \\
276 & 0,3175 & 0,3177
\end{tabular}


Dichte gesältigter Dämpfe und Flüssigkeiten.

Tabelle 12.

\begin{tabular}{c||c||c||c||c||c||c||c||c||c}
\hline \hline$T$ & $D$ & $d$ & $s$ & $s$ & $T$ & $D$ & $d$ & $S$ & $s$ \\
\hline \hline 190 & 0,690 & 0,020 & 1,449 & 50,0 & 240 & 0,629 & 0,038 & 1,590 & 26,3 \\
200 & 0,678 & 0,023 & 1,475 & 44,5 & 250 & 0,615 & 0,043 & 1,626 & 23,3 \\
210 & 0,666 & 0,026 & 1,501 & 38,5 & 260 & 0,600 & 0,048 & 1,667 & 20,8 \\
220 & 0,654 & 0,030 & 1,529 & 33,3 & 270 & 0,585 & 0,054 & 1,709 & 18,5 \\
230 & 0,642 & 0,034 & 1,557 & $\mathbf{2 9 , 4}$ & 280 & 0,570 & 0,060 & 1,754 & 16,7
\end{tabular}

V. Propionsäure.

Siedepunkt $140^{\circ}$; specifisches Gewicht bei $18^{\circ}: 1,005$.

Die Gleichung des Durchmessers ist:

$$
\Delta=0, \tilde{\mathbf{s}} 212-0,000605 t \text {. }
$$

Tabelle 13.

\begin{tabular}{c||c||c}
\hline$T$ & $\frac{d+D}{2}$ & $J$ \\
\hline \hline 190 & 0,4052 & 0,4053 \\
212 & 0,3928 & 0,3932 \\
237 & 0,3779 & 0,3781 \\
275 & 0,3539 & 0,3533
\end{tabular}

Tabelle 14.

\begin{tabular}{c||c|c||c||c||c||c||c||c||c}
\hline \hline$T$ & $D$ & $d$ & $S$ & $s$ & $T$ & $D$ & $d$ & $S$ & $s$ \\
\hline \hline 190 & 0,800 & 0,0103 & 1,250 & 97,2 & 240 & 0,722 & 0,029 & 1,383 & 34,4 \\
200 & 0,786 & 0,013 & 1,272 & $\mathbf{7 7 , 0}$ & $\mathbf{2 5 0}$ & 0,703 & 0,036 & 1,422 & 27,5 \\
210 & 0,772 & 0,016 & 1,295 & 62,5 & 260 & 0,682 & 0,044 & 1,466 & 22,7 \\
220 & 0,756 & 0,019 & 1,323 & $\mathbf{5 2 , 7}$ & $\mathbf{2 7 0}$ & 0,662 & 0,052 & 1,511 & 19,2 \\
230 & 0,740 & 0,023 & 1,351 & 43,5 & 280 & 0,642 & 0,060 & 1,558 & 16,7
\end{tabular}

VI. Normale Buttersäure.

Siedepunkt $162^{\circ}$; specifisches Gewicht bei $18^{\circ}: 0,969$.

Die Gleichung des Durchmessers ist:

$$
\Delta=0,4953-0,000528 t \text {. }
$$


Tabelle 15.

\begin{tabular}{c||c|c}
\hline \hline$T$ & $\frac{d+D}{2}$ & $\Delta$ \\
\hline \hline $190^{\circ}$ & 0,3950 & 0,3950 \\
212 & 0,3830 & 0,3834 \\
237 & 0,3690 & 0,3702 \\
275 & $0,3496^{\circ}$ & 0,3501
\end{tabular}

Tabelle 16.

\begin{tabular}{c||c||c||c||c||c||c||c||c||c}
\hline \hline$T$ & $D$ & $d$ & $S$ & $s$ & $T$ & $D$ & $d$ & $S$ & $s$ \\
\hline \hline $190^{\circ}$ & 0,790 & 0,0033 & 1,266 & 300 & $240^{\circ}$ & 0,713 & 0,020 & 1,403 & 50,0 \\
200 & 0,774 & 0,006 & 1,292 & 166 & 250 & 0,699 & 0,024 & 1,430 & $\mathbf{4 1 , 7}$ \\
210 & 0,758 & 0,009 & 1,319 & 111 & 260 & 0,685 & 0,028 & 1,460 & 34,3 \\
220 & 0,743 & 0,012 & 1,346 & 83,3 & 270 & 0,671 & 0,032 & 1,490 & 31,2 \\
230 & 0,728 & 0,016 & 1,373 & 62,5 & 280 & 0,657 & 0,036 & 1,520 & 27,8
\end{tabular}

Die Versuche bei $190^{\circ}$ liefern so grosse Werthe von $r$, dass dieselben nur geringe Ansprüche auf Genauigkeit machen können. Der Werth $d_{190}=0,0033$ ist kleiner als die normale Dampfdichte 0,00395; wenn dieser Werth aber auch etwas fehlerhaft sein kann, so lässt sich doch durch diesen Umstand allein das auffällig rasche Abnehmen der Dampfdichte mit der Temperatur nicht erklären.

\section{Isobuttersäure.}

Siedepunkt $153^{\circ}$; specifisches Gewicht bei $18^{\circ}: 0,959$.

Die Gleichung des Durchmessers wird

$$
\Delta=0,4965-0,000 \tilde{3} 73 t \text {. }
$$

Tabelle 17.

\begin{tabular}{c||c||c}
\hline$T$ & $\frac{D+d}{2}$ & $\Delta$ \\
\hline \hline $190^{\circ}$ & 0,3877 & 0,3876 \\
212 & 0,3760 & 0,3750 \\
237 & 0,3612 & 0,3607 \\
275 & 0,3396 & 0,3389
\end{tabular}


'Tabelle 18.

\begin{tabular}{|c|c|c|c|c|c|c|c|c|c|}
\hline$T$ & D & $d$ & $S$ & $s$ & $T$ & $D$ & $d$ & $S$ & $s$ \\
\hline $190^{\circ}$ & 0,762 & 0,013 & 1,311 & 77,7 & $240^{\circ}$ & 0,692 & 0,027 & 1,445 & 37,0 \\
\hline 200 & 0,750 & 0,014 & $\mathbf{1 , 3 3 3}$ & 71,4 & 250 & 0,673 & 0,033 & 1,486 & 30,3 \\
\hline 210 & 0,738 & $0,01 \mathfrak{\jmath}$ & 1,355 & 66,7 & 260 & 0,654 & 0,040 & 1,529 & 25,0 \\
\hline $2 \geq 0$ & 0,724 & 0,018 & 1,381 & 55,5 & 270 & 0,636 & 0,048 & 1,572 & 20,8 \\
\hline 230 & 0,708 & 0,022 & 1,412 & 45,4 & $2 s 0$ & 0,618 & 0,057 & 1,618 & $17, \overline{0}$ \\
\hline
\end{tabular}

Zur Theorie.

1. Die Beobachtungen gestatten eine directe Bestimmung der Constanten $a$ und $b$ der van der Waals'schen Zustandsgleichung:

$$
p=\frac{R T}{v-b}-\frac{a}{v^{2}} .
$$

Die Versuche liefern nämlich für das specifische Volumen $v$ zwei Werthe, $S$ für die Flüssigkeit, und $s$ für den Dampf, welche beide derselben Temperatur und demselben Druck entsprechen; also

$$
p=\frac{R T}{S-b}-\frac{a}{S^{2}}=\frac{R T}{s-b}-\frac{a}{s^{2}} .
$$

Ferner gilt für die Sättigungscurve die aus der Thermodynamik bekannte Gleichung:

$$
\int_{v_{1}}^{v_{2}} p d v=p_{1}\left(v_{2}-v_{1}\right) .
$$

Setzt man den aus Gleichung (I) genommenen Werth von $p$ hier ein und führt die Integration aus (wobei mit den bisher angewandten Bezeichnungen $v_{2}=S, v_{1}=s$ zu setzen ist), so erhält man

(II) $R T \log \cdot\left(\frac{S-b}{s-b}\right)+a \frac{s-s}{s \cdot \bar{S}}=(S-s)\left(\frac{R T}{s-b}-\frac{a}{s^{2}}\right)$.

Aus den beiden Gleichungen (I) und (II) ergiebt sich durch Elimination von $a$ :

$$
(s-b)(S-b) \log \cdot\left(\frac{S-b}{s-b}\right)=\frac{2 S \cdot s(S-s)}{s+s}-(S-s) b .
$$


Da diese Gleichung nicht direct auflösbar ist, so wurden ihre beiden Theile

$$
(s-b)(S-b) \log \frac{s-b}{s-b}=y
$$

und

$$
\frac{2 S s(S-s)}{S+s}-(S-s) b=y
$$

als Curven construirt und aus ihrem Schnitt der Werth von $b$ entnommen. Es ist dann ferner

$$
a=\frac{R T S S^{2} s^{2}}{(S-b)(s-b)(S+s)}
$$

wo

$$
R=\frac{\text { absolute Gasconstante }}{\text { Moleculargewicht }}=-\frac{81,49}{m} .
$$

Die bei jeder untersuchten Substanz für drei Temperaturen berechneten Werthe der Constanten $b$ und $a$ zeigen untenstehende Tabellen. Hieraus ergiebt sich, dass $b$ mit der Tem. peratur langsam wäclıst, während $a$ im allgemeinen abnimmt; nur bei Paraxylol wird auch a mit der Temperatur grösser.

Tabelle 19.

\begin{tabular}{c||c|c}
\hline \hline \multirow{2}{*}{$T$} & \multicolumn{2}{c}{ Toluol } \\
\cline { 2 - 3 } & $b$ & $a$ \\
\hline \hline \multirow{2}{*}{$200^{\circ}$} & 1,105 & 2456 \\
230 & 1,120 & 2365 \\
270 & $1,1 \neq 5$ & 2270
\end{tabular}

Tabelle 20.

\begin{tabular}{c||c|c||c|c||c|c}
\hline \hline \multirow{2}{*}{\multicolumn{1}{c||}{$T$}} & \multicolumn{2}{c||}{ Orthoxylol } & \multicolumn{2}{c||}{ Paraxylol } & \multicolumn{2}{c}{ Metaxylol } \\
\cline { 2 - 7 } & $b$ & $a$ & $b$ & $a$ & $b$ & $a$ \\
\hline \hline \multirow{2}{*}{$190^{\circ}$} & 1,097 & 2311 & 1,185 & 2143 & 1,110 & 2191 \\
230 & 1,120 & 2291 & 1,210 & 2226 & 1,125 & 2192 \\
270 & 1,135 & 2183 & 1,295 & 2469 & 1,150 & 2130
\end{tabular}


Tabelle 21.

\begin{tabular}{c||cc||c|c|c|c}
\hline \multirow{2}{*}{\multicolumn{1}{c||}{$T$}} & \multicolumn{2}{c||}{ Propionsiure } & \multicolumn{2}{c||}{$n$-Buttersäure } & \multicolumn{2}{c}{ Isobuttersäure } \\
\cline { 2 - 6 } & $b$ & $n$ & $b$ & $a$ & $b$ & $" 1$ \\
\hline $190^{\circ}$ & 0,990 & 3058 & 1,067 & 3451 & 1,040 & 2709 \\
230 & $1,0 \pm 5$ & 3081 & 1,080 & 3002 & 1,072 & 2715 \\
270 & 1,040 & $\mathbf{2 8 3 6}$ & 1,087 & 2752 & 1,100 & 2581
\end{tabular}

Die ,Constanten“ sind also nicht vollständig constant, $d$. h. die Zustandsgleichung entspricht den wirklichen Verhältnissen nur angenähert; dieses Resultat war rorauszusehen, da alle bisherigen Vergleiche der Theorie mit dem Versuch dasselbe ergeben haben. Die zablreichen anderen, von verschiedenen Autoren nach dem Muster ron van der Waals aufgestellten Zustandsgleichungen enthalten stets mebr als zwei Constanten, welche daher aus den hier besprochenen Versuchen mit Hülfe obiger Gleichungen nicht direct berechnet werden können. 2. Theorie des kritischen P'unktes. Die Dichten von Flüssigkeit und Dampf im Sättigungszustand stellen sich als Functionen der Temperatur in Form zweier Curven dur, welche nach der berrschenden Theorie an der kritischen Tempera-

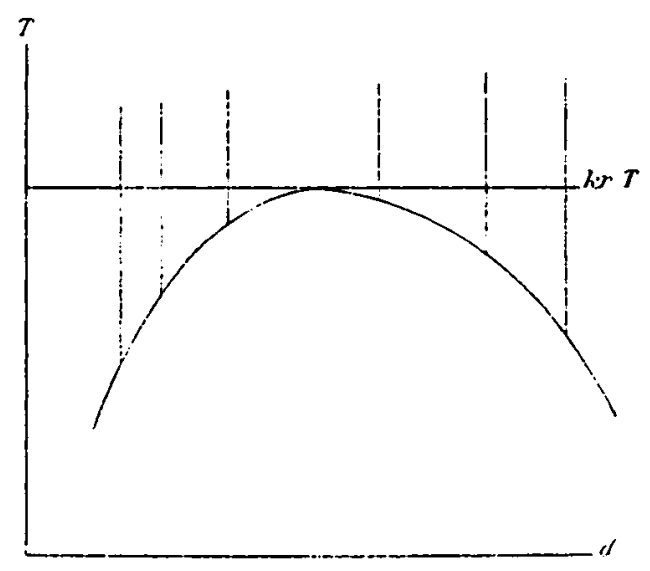

Fig. 4. tur ineinander übergehen sollen, indem sie dort ein Maximum der jetzt geschlossenen Curve bilden; dieser Maximalpunkt, an dem die Curve die Abscisse der kritischen Temperatur berührt, heisst der kritische Punkt, das ihm entsprechende Volumen das kritische Volumen.

Lässt man nun in einer geschlossenen Röhre, welche eine bestimmte Quantität Substanz theils als Flüssigkeit, theils als Dampf enthält, die Temperatur wachsen, so wird die gleich- 
zeitige Aenderung der Dichten in der Fig. 4 durch zwei Punkte dargestellt, die entlang den beiden Aesten der Sättigungscurve fortschreiten und solange anf ihr bleiben, als im Rohı Flüssigkeit und Dampf vorhanden sind. Dehnt sich die Flüssigkeit so stark aus, dass sie das ganze Rohr erfült, oder verdampft so viel Substanz, dass das Rohr nur mehr Dampf enthält, so hört die eine abbildende Linie auf, wäbrend die andere die Sättigungscurve verlässt und entlang der Ordinate weitergeht. Denn jetzt befindet sich eine homogene Substanz von unveränderlichem Gewicht im Rohr von unveränderlichem Volumen, die Dichte ist also constant.

Ist dagegen die Füllung so gewählt, dass beim Erreichen der kritischen Temperatur noch Flüssigkeit und Dampf im Rohr vorhanden sind, so müssen beide Aeste der Sättigungscurve vollständig bis zur kritischen Temperatur durchlaufen werden. Oberhalb dieser Grenze wird die Substanz plötzlich homogen, die abbildende Curve setzt sich also entlang einer Ordinate fort, die nur vom Verbältniss der verwandten Substanzmenge zum Rohrvolumen abbängig ist, also sehr verschiedene Lagen haben kann, da es in der Willkür des Beobachters liegt, wieviel Substanz das Rohr enthält.

Bezeichnen nun $d_{1}$ und $d_{2}$ die Dichten von Dampf und Flüssigkeit bei einer Temperatur unterhalb der kritischen, $v_{1}$ und $v_{2}$ die von beiden eingenommenen Volumentheile, $v$ das Gesammtvolumen des Rohres, $d$ die Dichte der homogen gewordenen Substanz bei einer Temperatur oberhalb der kritischen, endlich $p$ das Gewicht der Füllung, so ist

$$
\begin{aligned}
V d & =v_{1} d_{1}+v_{2} d_{2}=p, \\
d & =d_{1}+\frac{\left(d_{2}-d_{1}\right) v_{2}}{V},
\end{aligned}
$$

also

$$
d_{2} \geqq d \geqq d_{1},
$$

und da $d$ mit der Füllung willkürlich veränderlich ist:

$$
d_{2}>d_{1} \text {. }
$$

Hierbei kann $d_{2}$ und $d_{1}$ bei jeder Temperatur genommen werden, bei welcher noch Flüssigkeit und Dampf im Rohr vorhanden sind, d. h. bis zum Verschwinden des Meniscus. Bezeichnen also $\delta_{1}$ und $\delta_{2}$ die Dichten des gesättigten Dampfes 
und der Flüssigkeit an der kritischen Temperatur vor diesem Verschwinden der Trennungsfläche, so gilt auch hier:

$$
\delta_{2}>\delta_{1},
$$

d. h. die Dichten des gesättigten Dampfes und der Flüssigkeit bei der kritischen Temperatur sind nicht gleich; die beiden Sättigungscurven gehen nicht ineinander über, sondern treffen die kritische Temperatur in zwei verschiedenen Punkten, $A$ und $B$. (Vgl. Fig. 5.)

Aus Gleichung (I) ergeben sich für die kritische Temperatur die Grenzwerthe von $d$ :

$$
\begin{aligned}
& d=\delta_{1} \text { für } v_{2}=0, \\
& d=\delta_{3} \text { für } v_{3}=r, \text { d. h. } v_{1}=0,
\end{aligned}
$$

d. h. die Dichte des gesättigten Dampfes an der kritischen Temperatur ist gleich der Dichte der homogen gewordenen Substanz bei derjenigen Füllung, bei welcher bei der kritischen Temperatur gerade alle Flïssig. keit verdampft ist; die Dichte der Flüssigkeit unter Sättigungsdruck an der kritischen Tem. peratur ist gleich der Dichte der homogen gewordenen Substanz bei derjenigen Füllung, bei welcher bei der kritischen Temperatur die

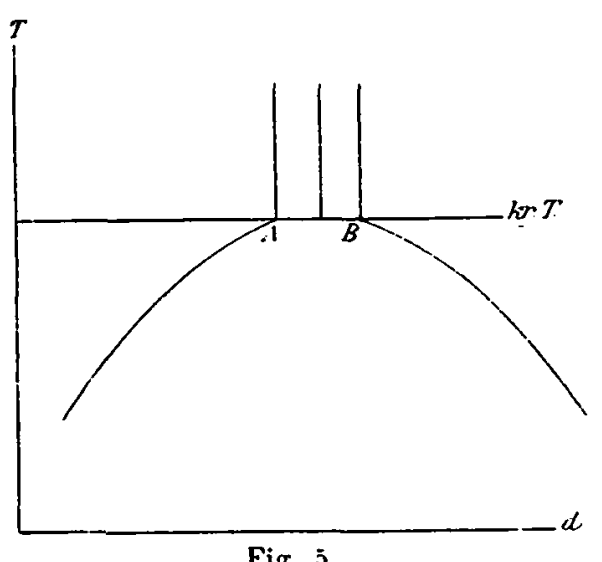

Fig. 5. Flüssigkeit das Rohr gerade ganz ausfüllt.

Der Deutlichkeit halber sei das Resultat obiger Entwickelung nochmals kurz zusammengefasst:

a) Oberhalb der kritischen Temperatur ist die Dichte der Substanz jedenfalls durch eine Ordinate dargestellt, deren Lage mit der Füllung willkürlich geändert werden kann, etwa durch $C c$ oder $D d$ oder etc.; die äussersten möglichen Lagen derselben seien $A a$ und $B b$. (Fig. 6.) 
b) Unterhalb der kritischen Temperatur sind die Dichten von Flüssigkeit und Dampf nach der herrschenden Theorie durch die Curven $l m K$ bez. $l^{\prime} n i^{\prime} K$ dargestellt.

c) Demgegenüber wird behauptet, der Verlauf dieser Curven sei $l^{\prime} m^{\prime} A$ bez. $l m B$.

Beim Ueberschreiten der kritischen Temperatur findet also in beiden Fällen ein plötzlicher Uebergang der Dichten statt, entweder von $K$ nach einem Punkt zwischen $A$ und $B$, etwa $C$, oder von $A$ und $B$ zugleich nach $C$. Während aber

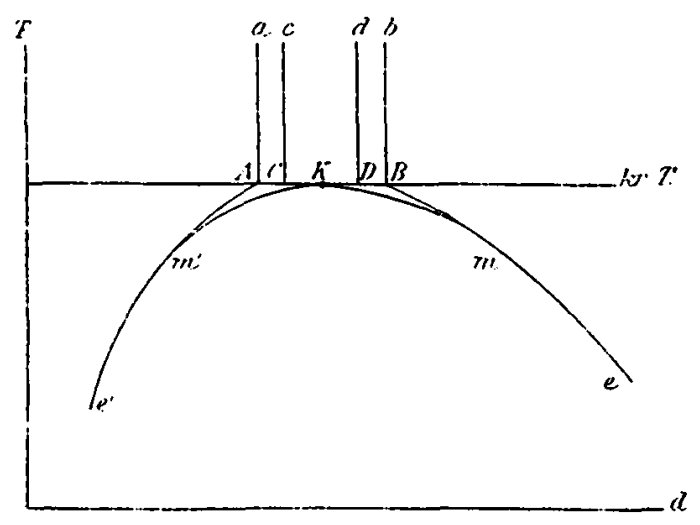

Fig. 6.

gegen diesen letzteren Weg von $A$ und $B$ zu $C$ nichts einzuwenden ist, erweist sich ein Uebergang $K C$ als unmöglich; denn in $K$ hätte die Substanz bereits eine einheitliche Dichte; eine Aenderung derselben im geschlossenen Rohr, d. b. bei constantem Volumen, würde eine Aenderung des Gewichtes in sich schliessen, ist daher unmöglich. Hierdurch ist bewiesen, dass die Sättigungscurve die kritische Temperatur nicht berührt, sondern ibre beiden Aeste sie in zwei verschiedenen Punkten treffen. Den diesen Punkten entsprechenden gesättigten Dichten $\delta_{1}$ und $\delta_{2}$ gebührt der Name , lritische Dichten", denn sie gebören als dritte Variable zur ,kritischen 'Temperatur" und zum „kritischen Druck“, der ja als Sättigungsdruck bei der kritischen Temperatur definirt ist.

Nach dem Gesetz ron Matthias ${ }^{1}$ ) wird die ,kritische

1) E. Matthias, Compt. rend. 115. p. 35. 1890. 
Dichte" als Schnittpunkt des Durchmessers mit der kritischen Temperatur bestimmt; diese Dichte

$$
\delta=-\frac{\delta_{1}+\frac{\delta_{2}}{2}}{2}
$$

entspricht offenbar dem Mittelpunkt von $A B$. Aus Gleichung(I)

$$
d=\frac{d_{1}+\left(d_{2}-d_{1}\right)}{V} \frac{v_{2}}{V}
$$

ersieht man, dass dieser Mittelwerth dann wirklich erreicht wird, wenn $v_{1}=v_{2}$ ist, d. h. der Meniscus gerade in der Mitte der Röhre verschwindet; dieses $\delta$ entspricht dann aber der homogen gewordenen Substau\%, keinem gesättigten Zustand und bildet daher mit der kritischen Temperatur und dem kritischen Druck keinen möglichen Zustand der Substanz; daher verdient $\delta$ auch nicht dell Namen einer "kritischen“ Dichte.

Jede experimentelle Bestimmung, welche den rerschwindenden Meniscus beobachtet, muss die Grössen $\delta_{1}$ und $\delta_{2}$ liefern. Denn da sie auf der Beobachtung ciner Trennungsfläche beruht, so setzt sie noch zwei getrennte Theile, Fliissigkeit und Dampf, voraus. Dennoch behaupten verschiedene Autoren auf Grund ihrer Versuche, die Dichten von Flüssigkeit und Dampf seien an der kritischen Temperatur gleich, so Amagat ${ }^{1}$ ) und Young ${ }^{2}$ ) bei Gelegenheit der Untersuchung von Pentan. Sie finden nämlich, dass bei den böchsten beobachteten Temperaturen beide Grössen so rasch aufeinander zustreben, dass die bei der kritischen Temperatur, welche einige Zehntel, ja einige Hundertstel Grade höher liegen soll, einander gleich würden. Offenbar braucht man die kritische Temperatur nur einige Hundertstel Grade niederer anzunehmen, um zu dem entgegengesetzten Resultat zu gelangen, und da die Erscheinungen im kritischen Zustand eine so genaue Bestimmung dieser Temperatur unmöglich mit Sicherheit ausführen lassen, so muss angesichts der logischen Unmöglichkeit, zu der er führt, dieser Scbluss der genannten Beobachter als unstatthaft angesehen

1) E. H. A magat, Compt. rend. 114. p. 1093 u. 1322. 1892: l'hỵsik. Revue 1. p. 37. 189:3.

2) S. Young, Journ. Chem. Soc. 71. 1. 446. 1897 . 
werden. Hier ist hervorzuheben, dass wenn auch wenige Bruchtheile eines Grades über der letzten beobachtbaren Temperatur die Dichtecurven ineinander übergehen würden, doch ihre thatsächlich gefundenen Endwerthe nicht in gleichem Maasse nahe aneinander liegen, da die Curven hier eben sebr flach verlaufen.

Battelli ${ }^{1}$ ) führt für die Gleichheit der kritischen Dichten einen theoretischen Grund an. Er sagt: In Gleichung

$$
u\left(T \frac{d p}{d T}-p\right)=0
$$

wo $u$ die Differenz der specifischen Volumina bedeutet, muss entweder $u$ oder der Klammerausdruck $=0$ sein; letzterer lässt sich berechnen und ist nicht $=0$; also ist $u=0$. Die angeführte Gleichung ist offenbar aus der bekannten Beziehung abgeleitet:

$$
r=T u \stackrel{d p}{d t},
$$

wo $r$ die Verdampfungswärme bedeutet. Zerlegt man dieselbe in die innere Verdampfungswärme o, welche die Energie der Molecüle vermehrt, und die äussere, welche die Ausdehnungsarbeit $p u$ leistet, und setzt an der kritischen Temperatur $\underline{\varphi}=0$, so erhält man die von Battelli angefübrte Gleichung. Offenbar liegt aber in der Voraussetzung $\rho=0$ bereits die Annabme, dass Dampf und Flüssigkeit identisch sind, der Schluss von Battelli ist also nicht stichhaltig.

Auch die Darstellung ron Stoletow ${ }^{2}$ ) in seiner Arbeit über den kritischen Zustand ist nicht einwurfsfrei. Er nimmt nämlich an, die abbildende Curve gehe nur dann durch den „kritischen Punkt:", wenn der Meniscus in der Mitte des Rohres verschwindet, während sie in jedem anderen Fall die kritische Temperatur an einem etwas seitwärts gelegenen Punkt überschreite. Da aber unterhalb der kritischen Temperatur stets Flüssigkeit und Dampf im gesättigten Zustand vorhanden sind, so ist hier ein Abweichen von der

1) A. Battelli, Physik. Revue 1. p. 264. 1892.

2) A. G. Stoletow, Physik. Revue 2. p. 44.1892. 
Sättigungscurve unmöglich, die Dichten sind durch die Temperatur allein vollständig bestimmt; erst oberhalb derselben werden sie von der Füllung abhängig, erst hier also kann die abbildende Curve sich verzweigen.

Eine vollständig hiervon verschiedene Auffassung rertritt Boltzmann in den „Vorlesungen über Gastheorie", II, p. 21: Er nimmt an, der Meniscus verschwinde uberhaupt nicht wirklich in der Röhre, sondern wandere auch bei der kritischen Temperatur stets nach einem Ende, wobei er jedoch so undeutlich würde, dass man ihn in den letzten Momenten nicht mehr sehen soll. Diese Darstellung ist der einzige Ausweg, bei welchem die Annahme einer kritischen Dichte bestehen kann, scheint aber den Versuchsresultaten direct zu widersprechen; denn aus den Angaben der verschiedenen Experimentatoren ergiebt sich, dass der Meniscus an einer ganz bestimmten Stelle im Rohr verschwindet.

Ueberträgt man die ausgeführten Betrachtungen in ein Coordinatensystem mit Druck und specifischem Volumen als Variablen, so ergiebt sich eine der früheren ganz ähnliche Figur: die beiden Grenzcurven vereinigen sich nicht, da für den kritischen Druck die Dichten nicht gleich sind, sie sind vielmehr durch das horizontale Stück der kritischen Isotherme verbunden. Die Curve hat also kein bestimmtes Maximum, die Annahme von van der Waals, dass am "kritischen“ Punkt$$
\frac{d p}{d v}=0
$$

$$
\frac{d^{2} p}{d p^{2}}=0
$$

sei, wird hinfällig; denn die erste Gleichung gilt für alle Punkte auf $A B$, die zweite für keinen, ein ,kritischer Punkt" existirt nicht. Van der Waals hat aus obigen Gleichungen den Zusammenhang der kritischen Grössen mit den Constanten seiner Zustandsgleichung abgeleitet, hieraus die reducirte Zustandsgleichung und schliesslich die Theorie der correspondirenden Zustände.

Die Grundlage dieser ganzen Entwickelung wird daher hinfällig; dagegen lässt sich aus dem vorhergehenden kein Schluss gegen die van der Waals'sche Anschauung ziehen, dass es möglich sei, die individuellen Constanten der Substanz 
478 R. von Hirsch. Dichte gesättigter Dämpfe und Flüssigkeiten. aus der Zustandsgleichung durch Einführung der kritischen Grössen zu entfernen; als solche müssen dann aber neben Temperatur und Druck die beiden kritischen Dichten von Flüssigkeit und Dampf angesehen werden. Erst wenn der Zusammenhang dieser Grössen mit den Constanten der (rich. tigen) Zustandsgleichung ermittelt ist, lässt sich die Frage entscheiden, ob eine „reducirte“ Zustandsgleichung und „correspondirende" Zustände existiren.

Es erübrigt mir, dem Leiter des Institutes, Hrn. Professor Dr. E. von Lommel, sowie Hrn. Professor Dr. L. Graetz, von dem die Anregung zu dieser Arbeit ausging, meinen Dank für ibre liebenswürdige Unterstützung auszusprechen.

(Eingegangen 7. Juni 1899.) 
I. Dichtecurven und Durchmesser für Toluol.

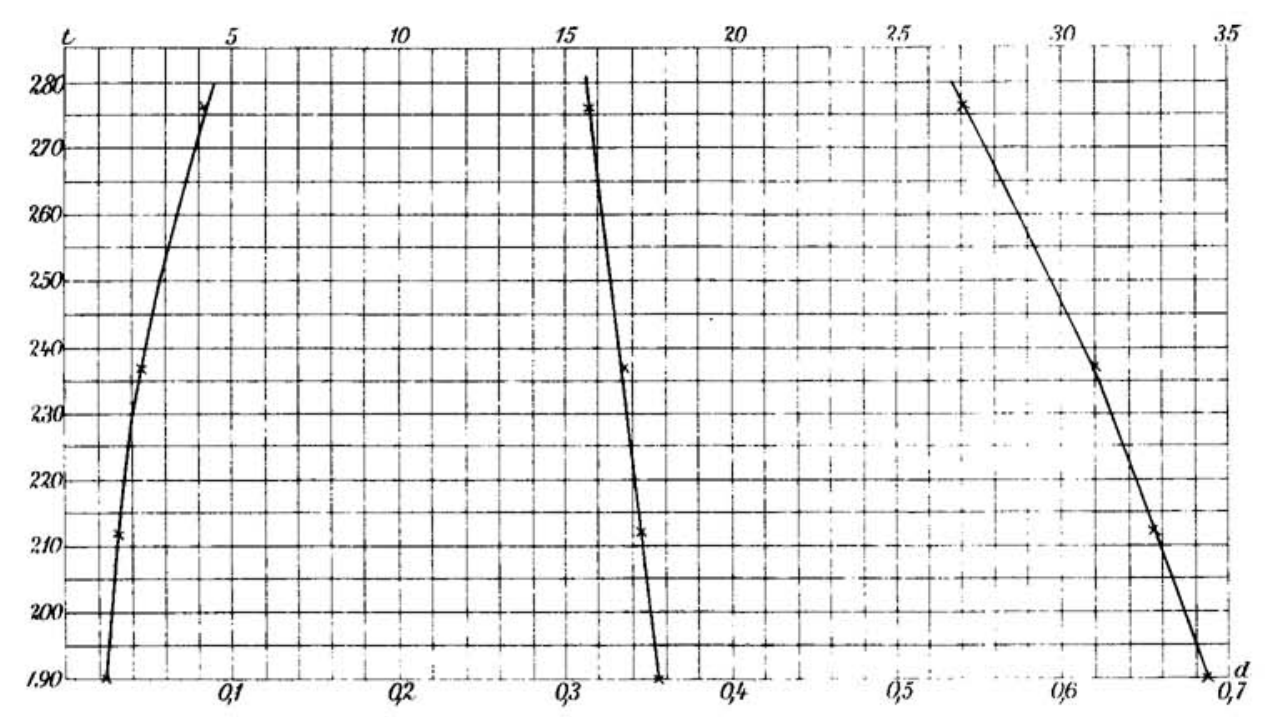

II. Dichtecurven und Durchmesser für Ortho-, Para- und Meta-Xyol.

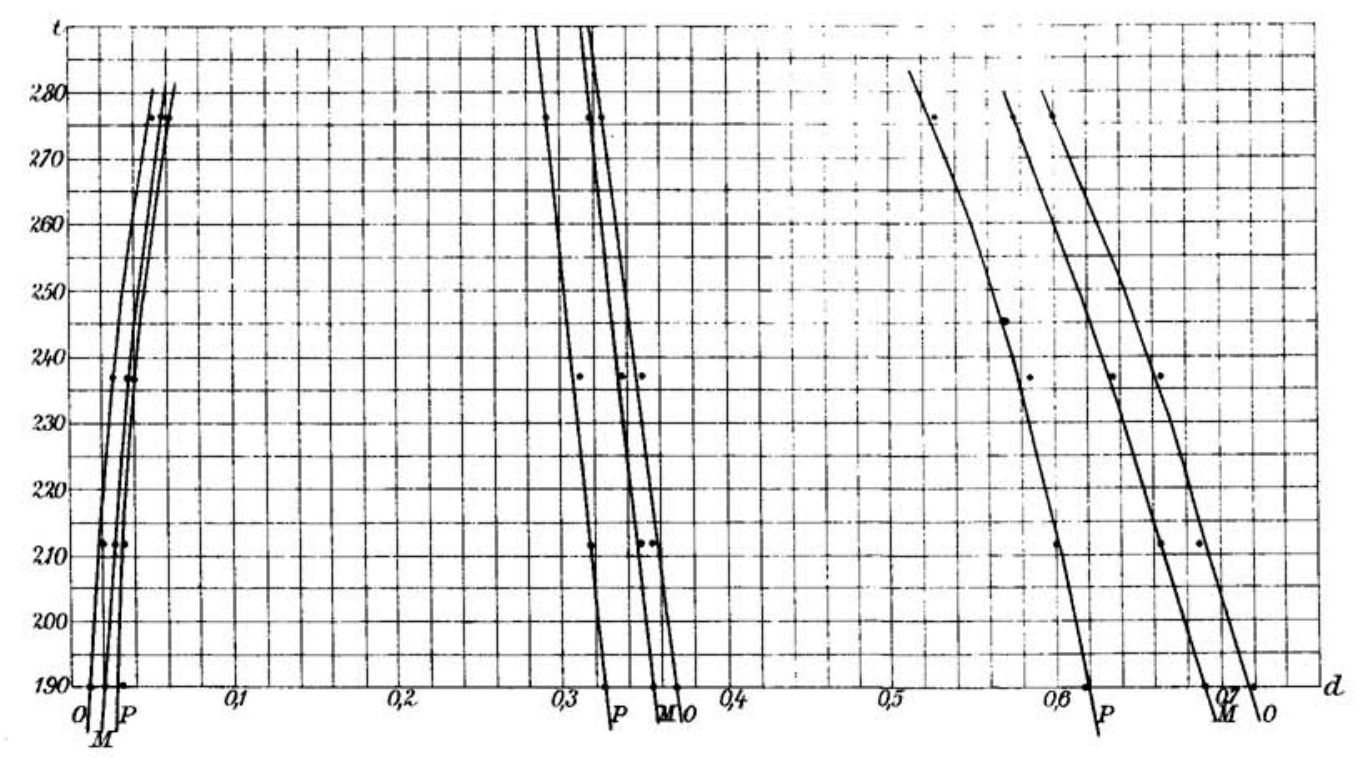

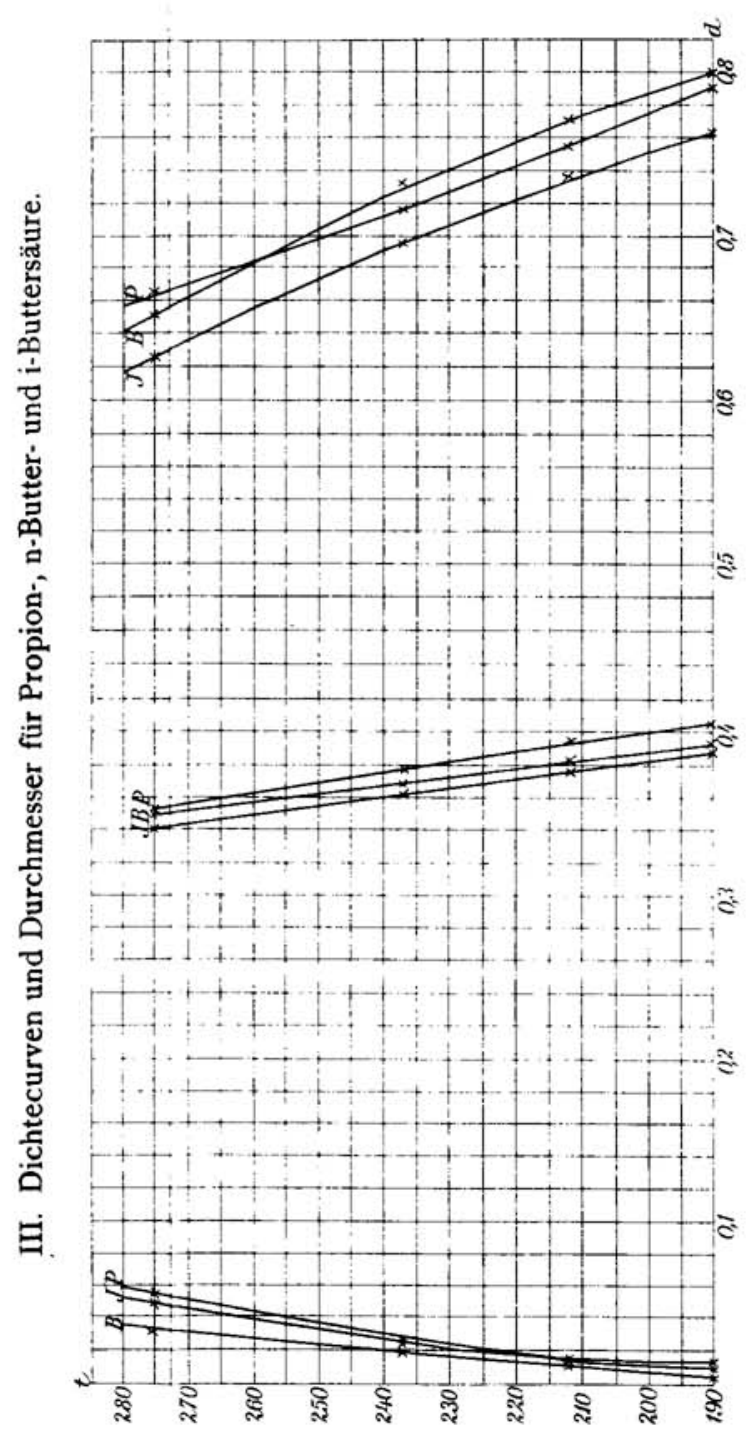

R.von Hirsch. 